\section{Relevance of Antigenicity of Candida Albicans Growth Phases to Diagnosis of Systemic Candidiasis}

\author{
E. G. V. EVANS, M. D. RICHARDSON, F. C. ODDS, \\ K. T. HOLLAND
}

British Medical fournal, 1973, 4, 86-87

\section{Summary}

The antigenicity of the two growth phases of Candida albicans has been compared by using crossed immunoelectrophoresis and double-diffusion techniques. Qualitative and quantitative differences in antigenic composition between the phases have been revealed and moreover a specific mycelial antigenic component demonstrated.

It is postulated that the use of specific mycelial antigens for routine diagnostic precipitin testing in patients with suspected systemic candidiasis would give more reliable results than at present obtained with yeast cell antigens.

\section{Introduction}

Systemic forms of candidiasis usually occur in debilitated patients, especially those on prolonged antibiotic or steroid therapy, and they are also seen with increasing frequency in patients who have undergone open-heart and transplantation surgery. They are serious infections, difficult to treat, and have an extremely poor prognosis. Speedy diagnosis of these rapidly fulminating infections is vital.

The precipitin test used in the diagnosis of systemic candidiasis is not entirely reliable. Antibodies to Candida species, both agglutinins and precipitins, have been found in patients in the absence of infection-for example, in those who have undergone open-heart surgery (Murray et al., 1969). These "false positive" results render the test subjective and could lead to errors in diagnosis.

Candida albicans has two growth phases, blastospores (yeast cells) and mycelium. Currently, a cytoplasmic antigen extracteed from the blastospore phase is used by most laboratories for the diagnostic precipitin test. C. albicans is thought to exist as a commensal in the blastospore phase only (Marples, 1960) but when causing an infection the mycelial phase is invariably produced (Gresham and Burns, 1960; Kozinn and Taschdjian, 1962). It is feasible to suggest that antigenic differences between the two phases may occur, as differences in chemical composition have been described (Dastidar, 1966; Chattaway et al. 1968; Dabrowa et al., 1970). Therefore, if it is assumed that the false positives are due to the presence of commensal blastospores, it would seem diagnostically more relevant to use a mycelial antigen for routine testing to detect antibodies in patients' serum against specific mycelial fractions. Hopefully this would be more reliable and would lead

\footnotetext{
Departments of Microbiology and Dermatology, University of Leeds and General Infirmary, Leeds LS1 3EX

E. G. V. EVANS, B.SC., Lecturer in Medical Mycology

M. D. RICHARDSON, B.SC., Research Assistant

F. C. ODDS, B.SC., PH.D., Research Fellow

K. T. HOLLAND, B.SC., PH.D., Lecturer in Microbiology
}

to an improvement in the immunological diagnosis of systemic candidiasis.

\section{Materials and Methods}

C. albicans serotype A was grown for 16 hours in neopeptonestarch broth (pH 7.5) (Chattaway et al, 1968) at $40^{\circ} \mathrm{C}$, which yielded pure mycelial growth with extensively developed branching filaments, and also at $30^{\circ} \mathrm{C}$, which gave pure blastospore growth. Cells were harvested by centrifuging $(1,500 \mathrm{~g})$ and washed extensively in phosphate-buffered saline (pH 7.2).

Antigens were prepared by disintegrating the cells with either a Braun MSK homogenizer (B. Braun, Melsungen, W. Germany) or an M.SE. Waring blender (M.S.E. Ltd., Crawley, Sussex) at 20,000 r.p.m. at $0^{\circ} \mathrm{C}$. Solid material was removed by high speed centrifugation $(17,000 \mathrm{~g})$ and the supernatant dialyzed against distilled water for 42 hours at $4^{\circ} \mathrm{C}$. Antigens were lyophilized and reconstituted in phosphatebuffered saline ( $\mathrm{pH} 7 \cdot 2)$ at optimal concentrations.

Antigens from the two growth phases were reacted against their homologous and heterologous antisera, prepared in rabbits from Braun extracts, using Laurell crossed immunoelectrophoresis (Laurell, 1965; Axelsen and Bock, 1972) and Ouchterlony double-diffusion techniques. With the Laurell technique the antigen in the well is first separated horizon-

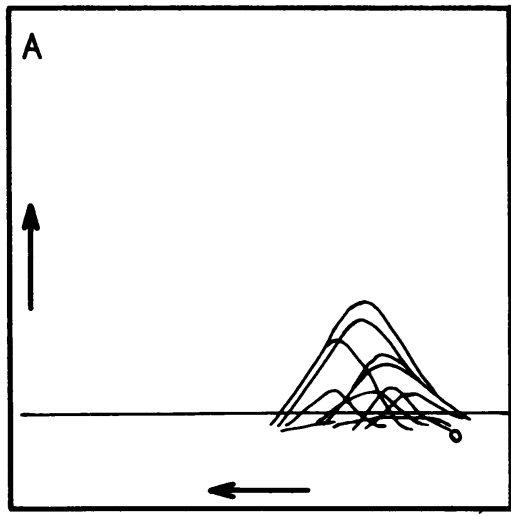

B

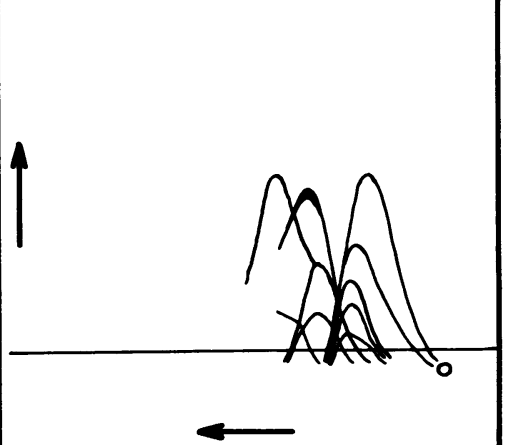
FIG. 1-Demonstration of Candida albicans precipitins by crossed immunoelectrophoresis (Laurell) of blastospore and mycelial antigens against homologous antisera. A. Mycelium cytoplasmic antigen (Braun). B. Blastospore cytoplasmic antigen (Braun). Agarose $1 \%, 0.12 \mathrm{M}$ sodium barbitone buffer pH $8 \cdot 6$. 
tally by electrophoresis and is then run vertically into the upper gell containing antiserum. The gel at $\mathrm{pH} 8.6$ prevents migration of immunoglobulins. On completion of electrophoresis, or after three days' double-diffusion at room temperature, the gels were washed for a total of 48 hours in a

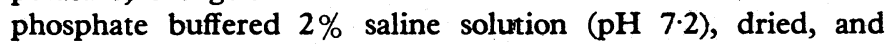
stained in 0.5\% Comassie Brilliant Blue R (G. T. Gurr, Ltd.).

\section{Results and Discussion}

The overall antigenic profile of the two growth phases when using the sensitive Laurell technique is different (fig. 1). The number of precipitation peaks and hence the total number of antigenic components is the same for both blastospores and mycelium. There are quantitative differences, as indicated by variation in peak areas, and a strong suggestion of qualitative differences between the phases as shown by positional differences of the peaks.

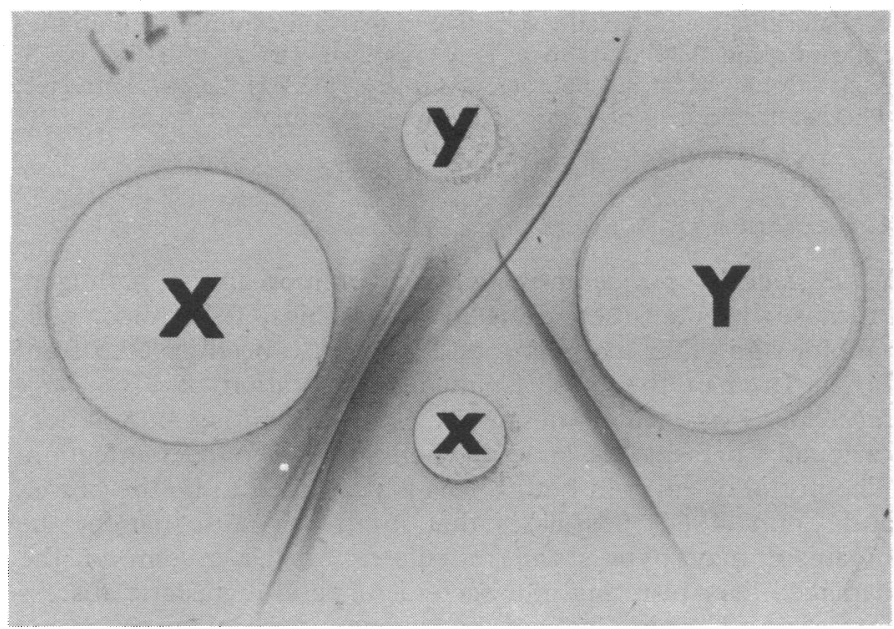

FIG. 2-Demonstration of Candida albicans precipitins by double-diffusion (Ouchterlony) of blastospore and mycelial antigens against homologous and heterologous antisera. X. Blastospore antiserum. Y. Mycelial antiserum. x. Blastospore antigen (M.S.E.) y. Mycelial antigen (M.S.E.). Immuno diffusion agar (Oxoid), sodium barbitone. Sodium acetate buffer pH 8.6.

The double-diffusion results confirm that qualitative antigenic differences exist between blastospores and mycelium. The reaction of the antigens of both phases to their homologous and heterologous antisera are shown in fig. 2. A reaction of non-identity between two component precipitin lines is clear when the antigens are reacted against the mycelial antiserum. This specific mycelial component is again shown in fig. 3

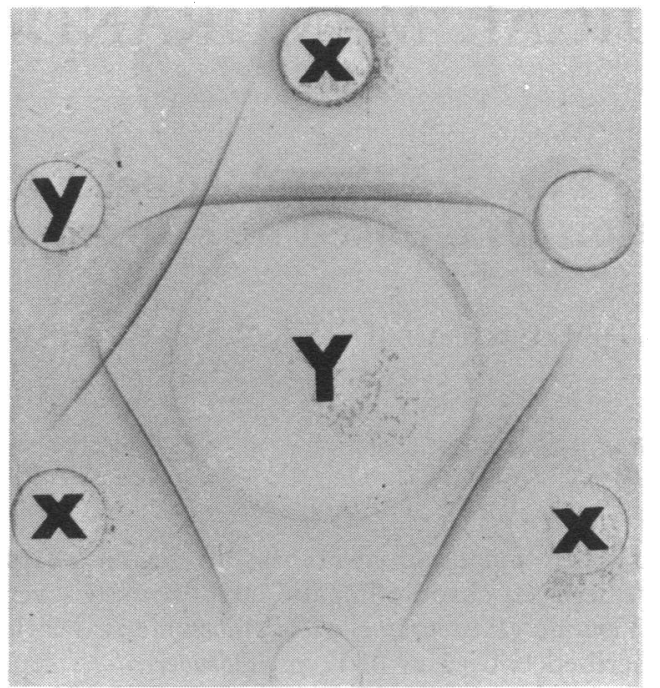

FIG. 3-Demonstration of Candida albicans precipitins by double-diffusion (Ouchterlony) of blastospore and mycelial antigens against mycelial antiserum. $\mathbf{Y}$. Mycelial antiserum. x. Blastospore antigen (M.S.E.) y. Mycelial antigen (M.S.E.). Conditions as in fig. 2 .

where the antigens of the two phases are reacted against mycelial antiserum. The complexity of the reaction partially obscures the result with the blastospore antiserum.

Work aimed at purification and fractionation of the antigenic components with concentration of antisera is in progress. This analysis may show further specific mycelial antigenic components, but the detection, so far, of at least one such component is encouraging. Hopefully this finding will eventually lead to an improvement in the immunological diagnosis of systemic candidiasis.

We are grateful to the Medical Research Council for financial support.

\section{References}

Axelsen, N. H., and Bock, E. (1972). Fournal of Immunological Methods, 1,

109.
Chattaway, F. W., Holmes, M. R., and Barlow, A. J. E. (1968). Fournal of General Microbiology, 51, 367.

Dabrowa, N., Howard, D. H., Landau, J. W., and Shecter, Y. (1970). Sabouraudia, 8, 163

Dastidar, S. G. (1966). Indian fournal of Experimental Biology, 4, 51.

Gresham, G. A and Burns, M. (1960). In Progress in the Biological Sciences in Relation to Dermatology, ed. A. Rook, p. 174. London, Cambridge University Press.

Kozinn, P. J., and Taschdjian, C. L. (1962). Pediatrics, 30, 71.

Laurell, C. B. (1965). Analytical Biochemistry, 10, 358.

Marples, M. J. (1960). Transactions of the Royal Society of Tropical Medicine and $\mathrm{H} y$ giene, 54, 166.

Murray, I. G., Buckley, H. R., and Turner, G. C. (1969). Fournal of Medical Microbiology, 2, 463 . 